\title{
THE STUDY OF PLASMA PARAMETER AND THE EFFECT OF EXPERIMENT SET UP MODIFICATION BY USING MODELLING SOFTWARE
}

\author{
Ersyzario Edo Yunata ${ }^{1 a *}$, Muhammad Ghufron ${ }^{2 b}$ \\ ${ }^{1}$ Department of Science and Technology, Airlangga University, Surabaya, INDONESIA. E-mail: \\ ersyzario.edo@fst.unair.ac.id ${ }^{\mathrm{a}}$ \\ ${ }^{2}$ Department of Physics, Brawijaya University, Malang, INDONESIA. E-mail: mghufron@ub.ac.id ${ }^{\mathrm{b}}$ \\ *Corresponding Author: ersyzario.edo@ fst.unair.ac.id \\ Submitted: $5^{\text {th }}$ December $2018 \quad$ Accepted: $21^{\text {st }}$ August 2019 \\ Published:30th November 2019
}

DOI: https://doi.org/10.22452/mjs.sp2019no3.1

\begin{abstract}
Plasma diagnosis has done by using Langmuir probe and software modeling. COMSOL and JMAG software were employed to characterize the electron density and identifying the set up effect in the plasma system. First, the COMSOL software was employed to simulate the behavior of electron density. The pressure and DC-bias voltage were varied during the simulation process. The DC bias voltage was varied in the range $-200 \mathrm{~V}$ to $-600 \mathrm{~V}$. The pressure was also varied in the range 30 to $80 \mathrm{~Pa}$. The high electron density was generated in the high DC-bias voltage and high pressure by using simulation process. Second, the Langmuir probe was also employed to measure the behavior of electron density inside the actual plasma chamber. The DC-bias voltage and pressure were also varied in the range $-350 \mathrm{~V}$ to $-650 \mathrm{~V}$ and $60 \mathrm{~Pa}$ to $120 \mathrm{~Pa}$, respectively. The experiment and modeling result were shown the same trend for the behavior of electron density. Third, the JMAG software was also utilized to characterize and modifying the set up in the plasma system. The electrode and DC bias was varied in the range 100 to $500 \mathrm{~V}$ and -100 to $-500 \mathrm{~V}$, respectively. The electric field distribution was concentrated in the electrode and DC-bias plate from the original set up. However, the electric field distribution was shifted to the center area after modification process. The modifications of experiment set up have provided the new way to confine the electric field. The high concentrated of electric field was effective to generate high plasma density.
\end{abstract}

Keywords: plasma, electron density, plasma modeling, electric field, and plasma diagnosis

\section{INTRODUCTION}

The plasma is considered to be the fourth state of matter, after the solid, liquids and gases and is generally visualized as an arc or discharge of bright fluorescent light. Physically, it is a partially or wholly ionized gas mixture containing reactive species, such as electrons, positive ions, negative ions, free radicals, excited or non-excited gas molecules and photon (Mandal et al., 2018). Nowadays, the plasma technology has been widely utilized in many sectors, such as medical (Dai et al., 2018), food packaging (Misra et al., 2019), aerospace (Kgoete et al., 2018), semiconductor (Fanelli et al., 2017), electrical sector (Zhao et al., 2017) and so on. In the industrial sector, there are two different modes of plasma such as thermal plasma or hot plasma and non-equilibrium or 
cold plasma. Thermal plasma is produced at high pressure $(>10 \mathrm{kPa})$ by means of direct (DC) or alternating current (AC) or radio frequency $(\mathrm{RF})$ or microwave sources with temperature around 2000-20000K. Nonequilibrium plasmas are low-pressure plasma characterized by high electron temperature and low ion and neutral temperatures (Samal et al., 2017). The study and characterization of plasma are needed with the purpose to obtain the optimum condition during application and plasma processing. There are many ways to characterize the plasma. Langmuir probe is one of the ways to measure the plasma density (Meshcheryakova et al., 2015). Optical emission spectroscopy (OES) is effective to describe the population in the plasma (Li et al., 2018). Both diagnostics are direct measurement of the plasma. However, there is an indirect method to characterize the plasma, that is through modeling. The modeling simulation provides more understanding of the processes in the plasma (Brezmes et al., 2014).

In the present study, two modeling software and Langmuir probe device were employed to study the plasma. The COMSOL software and Langmuir probe were utilized to characterize the effect of electron density with pressure and DC-bias voltage. Furthermore, the JMAG software was employed to describe the electric field distribution in the present set up. A modification of experiment set up was also employed with the purpose to get the optimum condition and better result for plasma processing. The modeling result was compared with the previous Langmuir probe diagnostic results (Yunata et al., 2013).

\section{EXPERIMENTAL PROCEDURE}

The plasma modeling consisted of several parts. First, the COMSOL software was utilized to simulate the effect of pressure and DC-bias voltage variation in the electron density. Second, the Langmuir probe was utilized to measure the plasma density inside the chamber. Third, the JMAG software was employed to characterize the effect of electric field in the experiment set up.

\subsection{Plasma modelling by using COMSOL software}

The one dimension plasma modelling was built to simulate the electron density behaviour in the COMSOL software. The effect of pressure and DC-bias voltage in the electron density was the main discussion in this study. The schematic of plasma modelling is depicted in figure 1 . The chamber was modelled with rectangular form. The chamber was grounded similar to the original plasma machine condition in the laboratory. The cathode was also modelled with rectangular form. The cathode was biased with negative voltage. In the DC-bias voltage variation, the voltage was varied in the range of -200 to $-600 \mathrm{~V}$. Next, the range of low gas pressure from 30 to $80 \mathrm{~Pa}$ was chosen to characterize its effect in the electron density in plasma. The secondary electrons were chosen as a mechanism to generate plasma. The plasma was generated inside the chamber. The detail of simulation condition was depicted in the table 
Table 1. Plasma simulation condition by using COMSOL software.

\begin{tabular}{cc}
\hline Parameters & \\
\hline DC-bias voltage & -200 to $-600 \mathrm{~V}$ \\
Pressure & 30 to $80 \mathrm{~Pa}$ \\
Temperature & $400 \mathrm{~K}$ \\
Gas & Argon \\
\hline
\end{tabular}

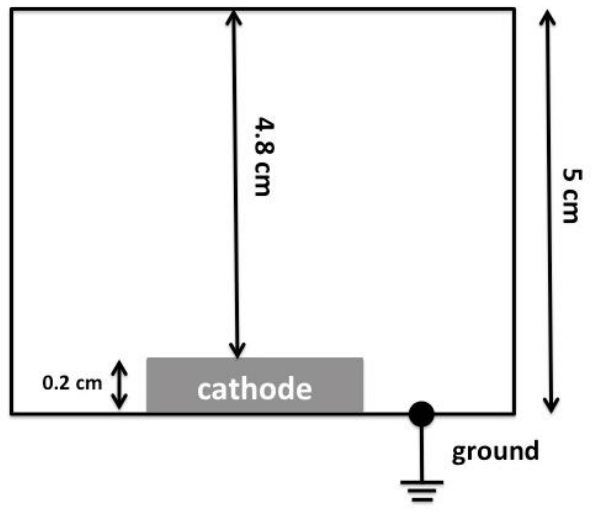

Figure 1. The schematic plasma modelling by using COMSOL software.

\subsection{Langmuir Probe measurement process}

Plasma parameter was measured with a single Langmuir probe analysis (Impedans ALP System). The schematic of the plasma system with Langmuir probe is depicted in figure 2. Details of probe specification are as follows: probe radius of $3.5 \times 10^{-4} \mathrm{~m}$, length of $0.01 \mathrm{~m}$ and resistance of $36 \mathrm{Ohm}$. The Langmuir probe was placed in the center of the chamber during the plasma processing. The electron density was measured at varying DC-bias and pressure. Then, the measured data were transferred and stored in the computer.

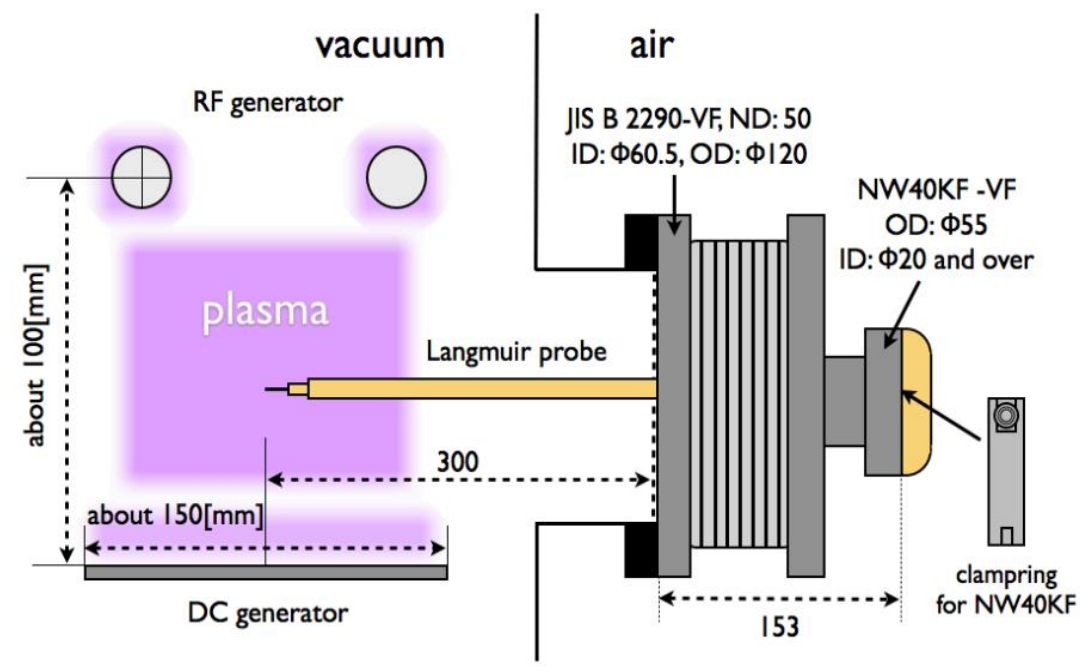

Figure 2. The schematic set up of Langmuir probe in plasma system 


\subsection{Plasma modelling by using JMAG software}

The JMAG software was utilized to characterize the electric field distribution in the plasma system. The chamber was modelled with big circle form. The chamber was grounded as the actual plasma machine condition in laboratory. The electrode was also modelled with small circular form. The electrode was set with positive voltage from 100 to $500 \mathrm{~V}$. The DC bias plate was modelled with rectangular form. The DCbias plate was set with negative voltage. The DC-bias voltage was varied in the range -100 to $-500 \mathrm{~V}$. The schematic of JMAG simulation is depicted in figure 3. The voltage variation was done in the electrode and DC bias plate. The electric field was generated inside the chamber in this modelling result. The distribution of electric field was characterized in each variation. Modification to the experiment set up was also employed in this process in which the model of a metal conductor was inserted in the area between electrode and DC-bias plate. The simulation result was studied and applied in the real plasma processing system in the laboratory.

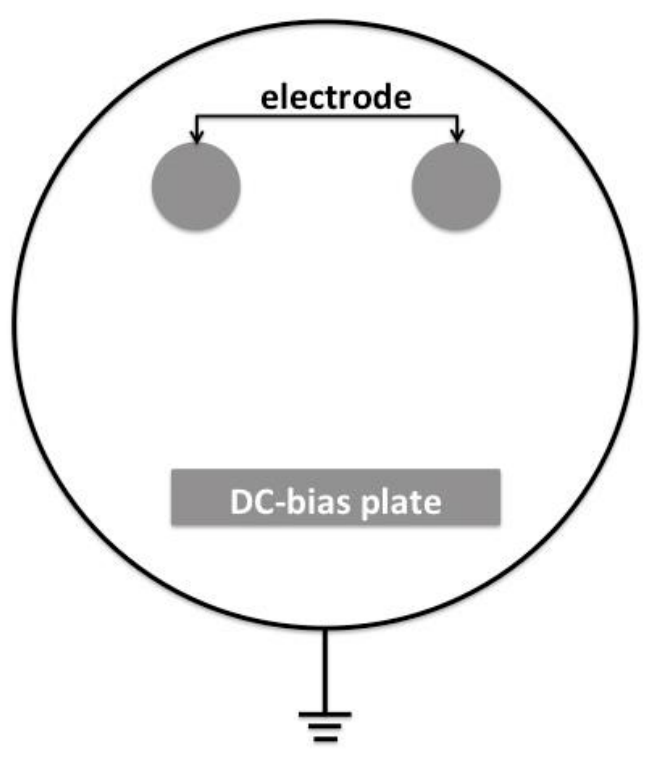

Figure 3. The schematic plasma modelling by using JMAG software.

\section{RESULT AND DISCUSSION}

\subsection{The behaviour of electron density}

The COMSOL software was employed to simulate the electron density behavior with variation of DC-bias and pressure. First, the pressure variation was simulated in the COMSOL software. The pressure was varied in the range of 30 to 80 $\mathrm{Pa}$. The result of pressure variation is depicted in figure 4 . The electron density increased from a low value of $1 \times 10^{18} \mathrm{~m}^{-3}$ with increasing pressure to a high value of $3 \times 10^{18} \mathrm{~m}^{-3}$. Electron density is related to ionization process inside the chamber. At low pressure, fewer gas molecules were introduced to the chamber and less collisions occur. The ionization process occur from electron collision with the gas molecules. Ions and secondary electrons resulted in this process. Fewer gas molecules produced low 
electron density. At high pressure, higher percentage of collision process occurred. As a consequence, a lot of electrons are produced through the ionization collision process and high electron density resulted.

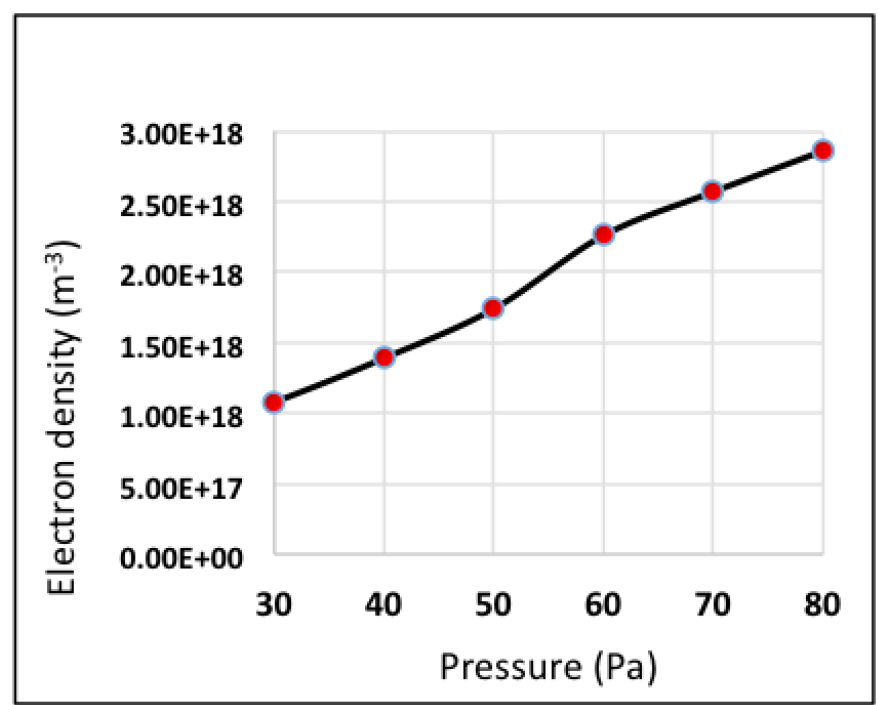

Figure 4. The effect of pressure variation in electron density

The DC-bias variation was also done in this simulation program. The effect of DC-bias variation is depicted in the figure 5 . At low DC bias voltage, the electron density was $9.55 \times 10^{17} \mathrm{~m}^{-3}$. The electron density increased with the increasing of DC-bias voltage. In the simulation, the DC-bias voltage indicates the quantity of electrons inside the chamber. The high DC-bias accelerates the ionization process and increases the percentage of ionization process. As a result, high electron densities were produced in this condition

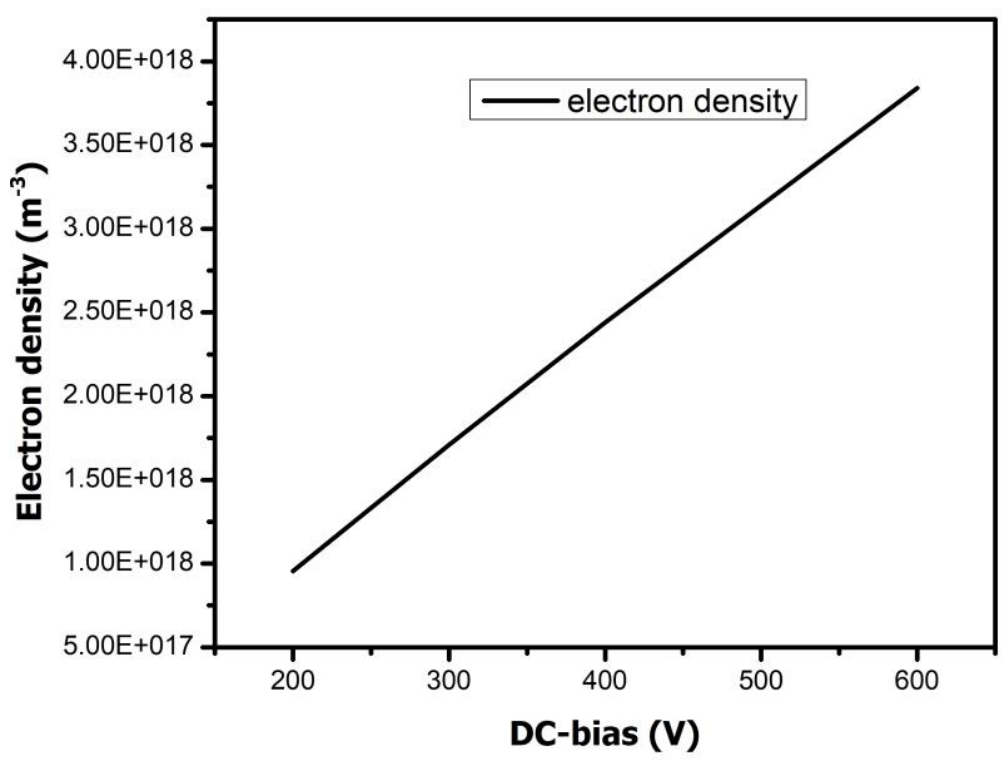

Figure 5. The effect of DC-bias variation in electron density 
The result of the modelling program was compared with the measurement result from Langmuir probe in a previous study. The Langmuir probe was utilized to measure the electron density inside the chamber. The measurement result is shown in the figure 6 . The Langmuir probe measurement result indicates the same trend with the simulation result. The pressure variation indicates high electron density was produced in higher pressure. The DC-bias variation showed the same result with the simulation result.

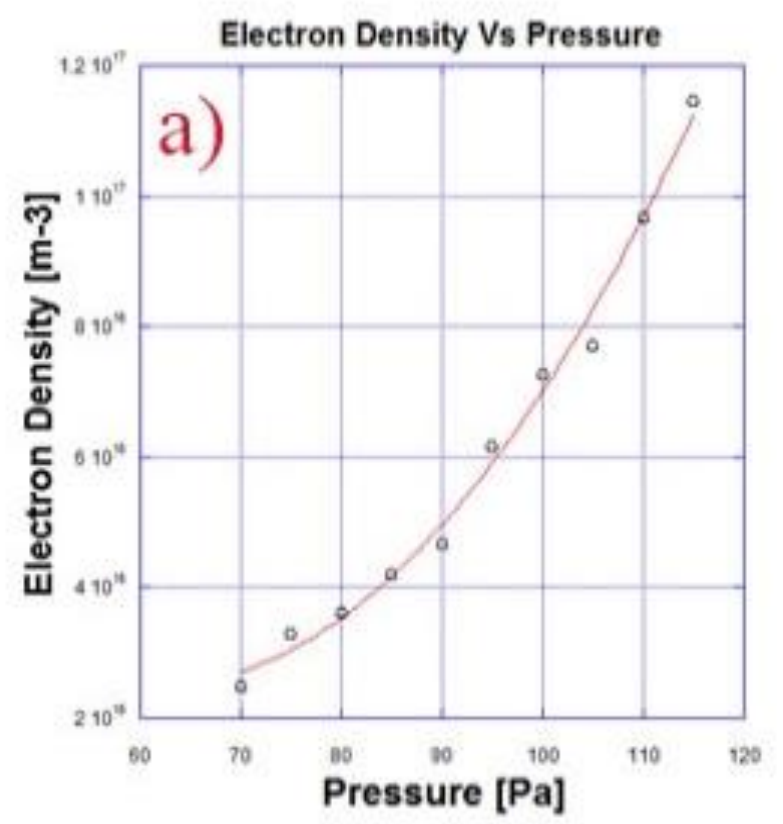

Basically, the DC-bias is utilized to accelerate the ion bombardment to the specimen in the experiment condition. In this case, the DC-bias increased the production of free electrons inside the chamber. The collisions of electrons and gas molecules also increased inside the chamber. As a result high electron density is produced in the high DC bias voltage. The simulation results are proposed to enhance the understanding of diagnosis by using measurement devices.

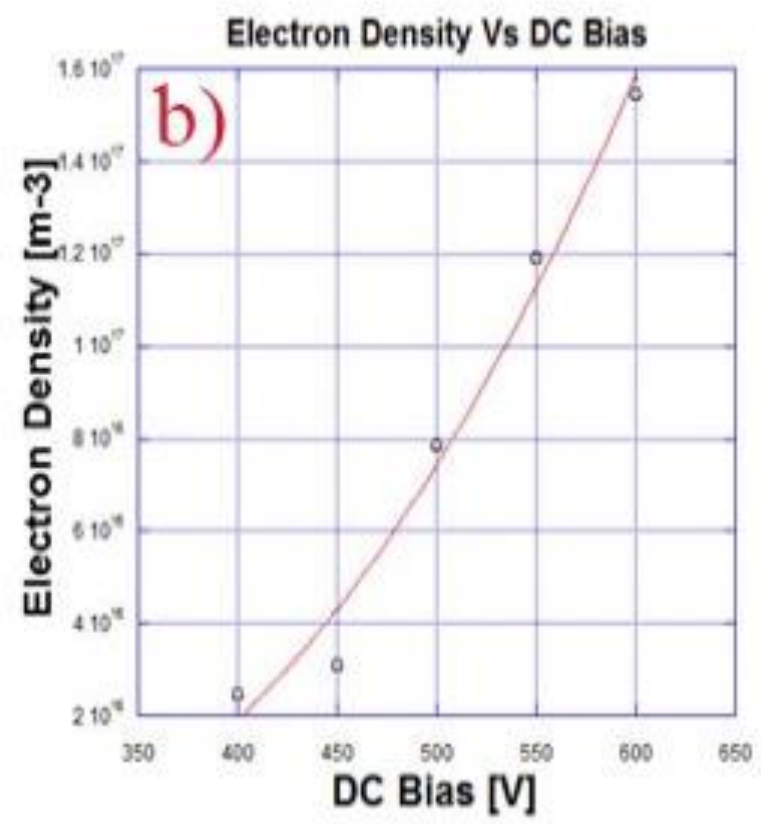

Figure 6. The pressure and DC bias variation results by using Langmuir probe

\subsection{The effect of electric field distribution in the experiment set up}

The JMAG software was employed to characterize the electric field distribution in the plasma system. The experiment set up has influence in the distribution of electric field. The voltage variations in the electrode and DC-bias have employed to characterize the electric field distribution. The results of electric field distribution in the present set up are shown in the figure 7 . The green color indicates the main distribution of electric field in the simulation result. 

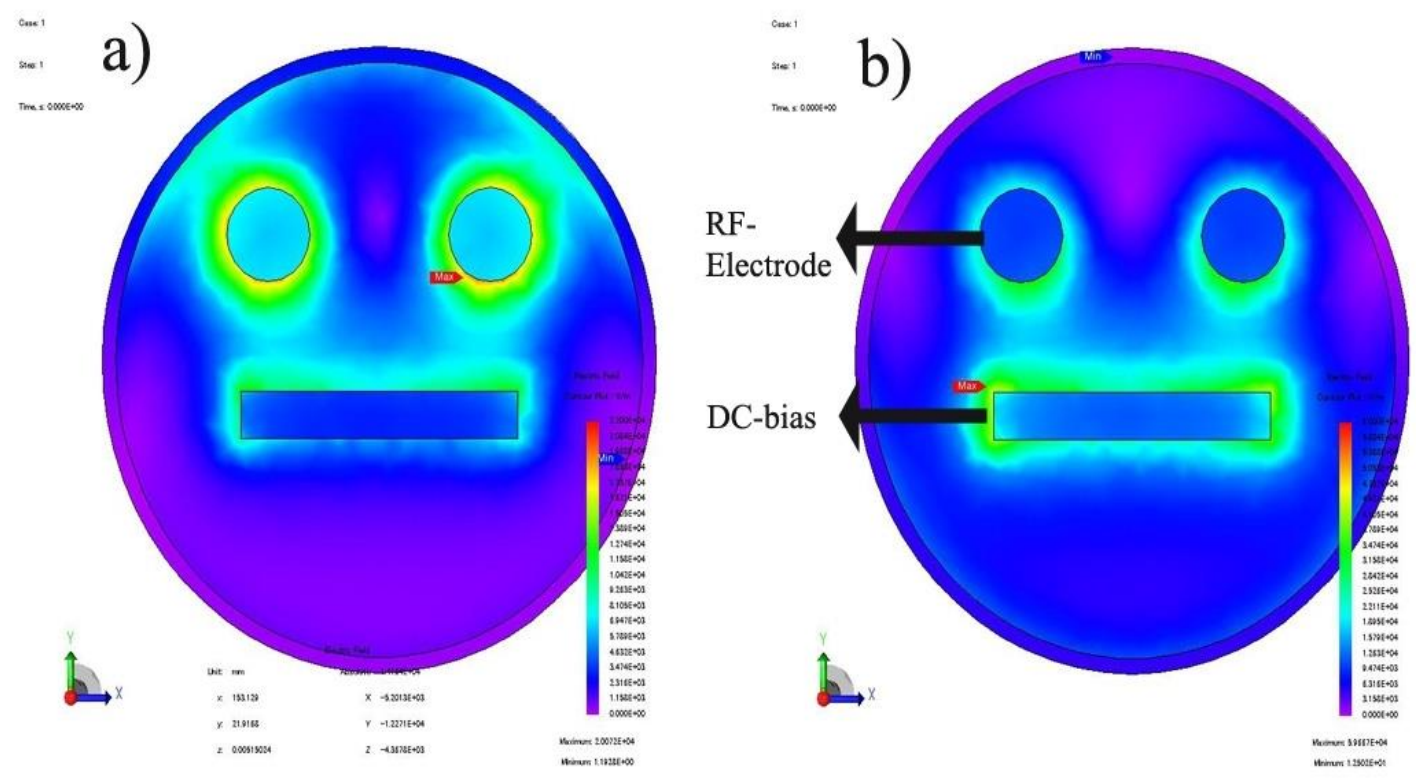

Figure 7. The electric field distribution in a) high RF voltage and low DC-bias voltage, b) low RF voltage and high DC bias voltage

Figure $7 \mathrm{a}$ represents the electrode at $250 \mathrm{~V}$ and the DC bias plate (-)100 V. The strong electric fields are concentrated in the electrode area. On the other hand, the DCbias plate has weak concentrate of electric field. The main distribution of electric field is shown with the green color. However, the figure $7 \mathrm{~b}$ represents a different electric field distribution result. The strong electric fields have shifted down to be close the sides of the DC bias plate due to the low voltage in the electrode and high voltage in the DC-bias plate. Both of the results have described the distribution of electric field in the present set up. The simulation result showed that the electric field direction depends on the high voltage which was applied in the electrode or DC-bias plate and is not uniformly distributed. In plasma processing the specimen are located on the DC-bias plate, modification of experiment set up has to be employed to solve this problem.

The new set up has been built to enhance the electric field distribution. The present set up has been modified by inserting a conductor as depicted in figure 8. This conductor is a hollow cathode, made from stainless steel. The hollow cathode is proposed to localize the glow discharge plasma and generate high electric field. The simulation result in figure $8 \mathrm{a}$ indicates a different distribution result from the previous result. The bright colour indicated in the area near the hollow cathode represents strong electric field. The bright colour almost covers the model of hollow cathode in the simulated result. This new diagnosis result has been applied in the present experiment in the laboratory as depicted in figure $8 \mathrm{~b}$. The glow discharge of hollow cathode plasma is concentrated inside the hollow tube area. The localized plasma is indicated with the strong and bright colour. The hollow cathode plasma provides high plasma density and effective for plasma etching (Yunata et al., 2016) and also plasma nitriding (Aizawa et al., 2017). 

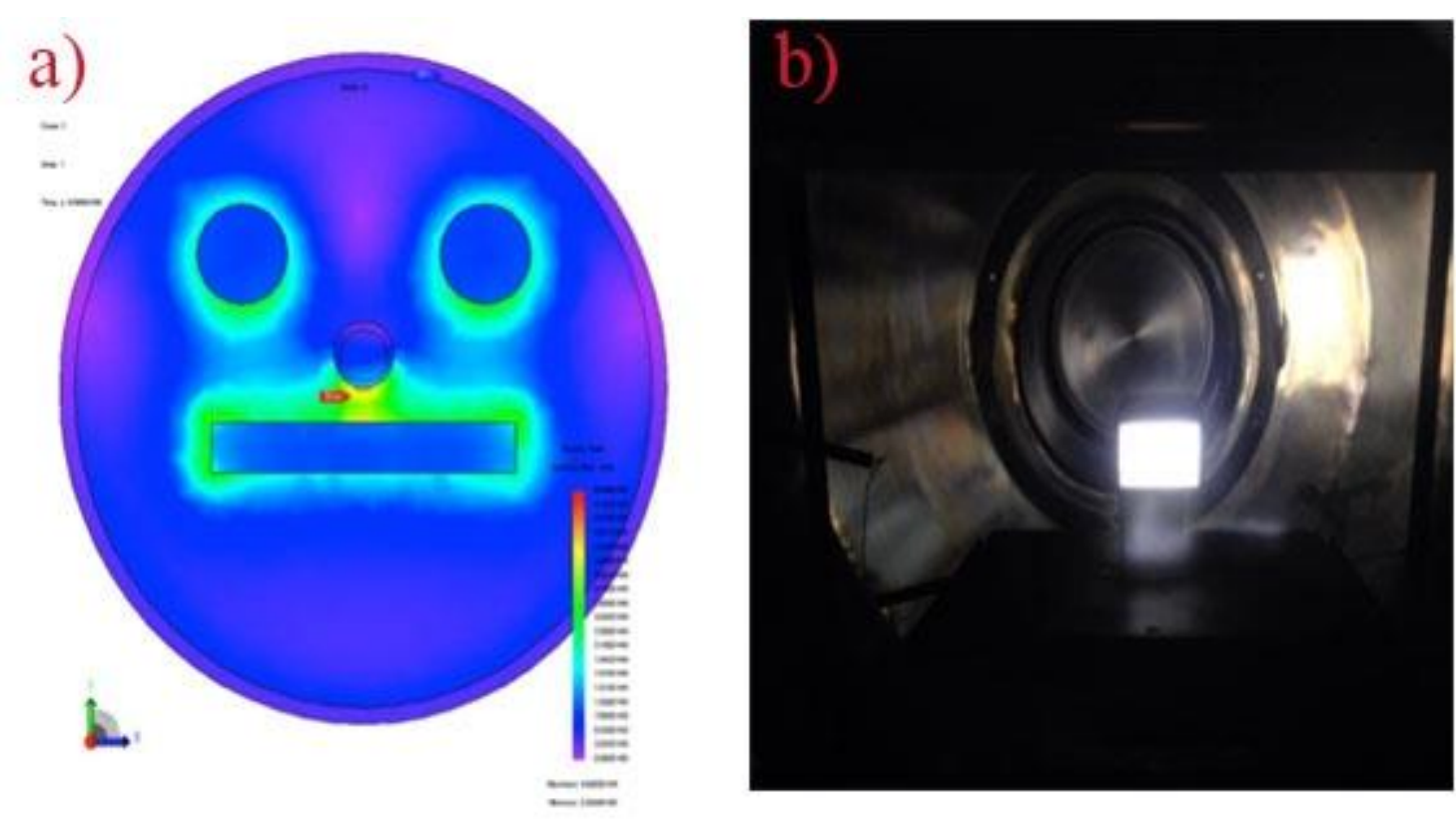

Figure 8. a) the electric field distribution in the new set up with hollow cathode, b) the glow discharge of hollow cathode plasma

\section{CONCLUSION}

The behaviour of electron density in the variation of DC-bias voltage and pressure was modelled with COMSOL software. The electron density increased with the increasing of DC-bias voltage and pressure. The high electron density indicated high percentage of ionization process inside the chamber. Electric field distribution was simulated using JMAG sofware. The simulation result showed the electric field distribution to be concentrated nonuniformly in the electrode or the DC-bias plate. A new set up with hollow cathode provided a solution to distribute the concentrated the electric field to cover over the surface of the DC-bias plate more evenly and in the centre between the hollow cathode and the DC-bias plate. High plasma density could be produced by using hollow cathode system.

\section{ACKNOWLEDGEMENT}

The authors would like to express their gratitude to Prof. Tatsuhiko Aizawa (Aizawa Sensei Laboratory) and Dr. Hijikata (Shibaura IT Japan) for their help in the experiment and modeling program.

\section{REFERENCES}

Aizawa T., et al.(2017). Fine piercing of electromagnetic steel sheets by plasma-nitrided tools, Procedia Engineering 247:1027-1032.

Brezmes A O., et al .(2014) Simulation of inductively coupled plasma with applied bias voltage using COMSOL, Vacuum. 109:52-60.

Dai X., et al.(2018). The emerging role of gas plasma in Oncotheraphy, Biotechnology. 36:1183-1198. 
Fanelli F., et al.(2017). Atmospheric pressure non-equilibrium plasma jet technology: general features, specificities and applications in surface processing material, Surface \& Coatings Technology 322 :174201.

Kgoete F. M., et al.(2018) Study on properties and microstructure of $\mathrm{Ti}-$ $\mathrm{Si}-\mathrm{N}$ coatings deposited in deep holes, Jounal of Alloys and compounds 769: 817-823.

Li C., et al.(2018). Optical emission spectroscopy study for pulsed frequency powered atmospheric $\mathrm{He}$ plasma. Surface \& Coating Technology 353:316-323.

Mandal R., et al.(2018) Recent developments in cold plasma decontamination technology in the food industry, Food Science \& Technology 80:93-103.

Meshcheryakova E., et al.(2015) Langmuir probe diagnostic of low-pressure inductively coupled argon plasma in magnetic field, Physics Procedia 71:121-126.

Misra N N., et al.(2019) In-package cold plasma technologies, Journal of Food Engineering 244:21-31.

Samal S., et al.(2017) Thermal plasma technology: The prospective future in material processing, Journal of Cleaner Production 142: 3131-3150.

Yunata E E., et al.(2013) Quantitative argon plasma characterization by Langmuir probe methode. The $7^{\text {th }}$ SEATUC Symposium ITB Indonesia 1-5.

Yunata E E., et al.(2016) Micro-grooving into thick CVD diamond films via hollow-cathode oxygen plasma etching, Manufacturing Letters 8:1620.

Zhao S., et al.(2017) Analysis of plasma oscillations by electrical detection in Nd:YAG laser welding, Journal of Material Processing Tech 249: 479489. 